\title{
A Simulação e a Videoconferência no Ensino de Enfermagem
}

\author{
Alessandra Mazzo', José Carlos A. Martins², Rui C. Negrão Baptista², Simone Godoy¹, Verónica \\ R. Dias Coutinho², Carlos A. Seixas¹, Isabel A. Costa Mendes¹, Juliana Constantino Franzon¹
}

${ }^{1}$ Escola de Enfermagem de Ribeirão Preto da Universidade de São Paulo.

${ }^{2}$ Escola Superior de Enfermagem de Coimbra, Portugal.

* Autora para correspondência: amazzo@eerp.usp.br

\section{RESUMO}

O objetivo deste estudo foi comparar os resultados da aprendizagem de estudantes de graduação em enfermagem que utilizaram estratégias de ensino simulado de baixa fidelidade com os resultados dos que usaram videoconferência do ensino simulado de alta fidelidade. Trata-se de um trabalho experimental realizado com graduandos de enfermagem do Brasil, os quais foram divididos em dois grupos e submetidos a diferentes estratégias de educação a distância: Ambiente Virtual de Aprendizagem (AVA) e videoconferência. Os dados foram coletados por meio de questionários e avaliação teórica e teórico-prática. Em linhas gerais, foram comparados os dois grupos de estudantes, os quais haviam sido distribuídos aleatoriamente e submetidos ao ensino simulado de baixa fidelidade (17) e à videoconferência do treino simulado de alta fidelidade e seu respectivo debriefing (27). O estudo foi realizado seguindo os preceitos éticos. Utilizou-se o Termo de Consentimento Livre e Esclarecido. Os estudantes relataram que o AVA e a videoconferência auxiliaram no seu aprendizado. Observou-se ainda que foi melhor o desempenho da aprendizagem dos alunos submetidos à simulação de baixa fidelidade do que o daqueles que assistiram à videoconferência do treino simulado de alta fidelidade e seu respectivo debriefing: Conclui-se que o ensino simulado é uma estratégia de ensino eficaz que promove de modo efetivo a participação do aluno como ator de seu aprendizado.

Palavras-chave: Enfermagem; Simulação; Educação a Distância; Videoconferência; Educação.

\begin{abstract}
The objective of this study was to compare the learning outcomes of undergraduate nursing students who used simulated low fidelity teaching strategies with the results of those who used high fidelity simulated teaching videoconference. This is an experimental study carried out with undergraduates nursing students from Brazil which were divided into two groups and submitted to different distance education strategies: Virtual Learning Environment (AVA) and videoconference. The data were collected through questionnaires and theoretical and theoretical-practical evaluation. In general terms, this work compared the two groups of students, which had been randomly distributed and submitted to simulated low fidelity teaching (17) and to the videoconference of the simulated high fidelity training and its respective debriefing (27). The present study was carried out following the ethical precepts. The Free and Informed Consent Term was used. The students reported that VLE and video conferencing aided their learning. It was also observed that the learning performance of the students submitted to the simulation of low fidelity was better than those who attended the videoconference of the simulated high fidelity training and its respective debriefing. It is concluded that the simulated teaching is an effective teaching strategy that promotes the participation of the student as an actor of his learning.
\end{abstract}

Keywords: Nursing; Simulation; Distance Learning; Videoconferencing; Education.

\section{Introdução}

Os processos educativos percorrem da potência ao ato da realização, visando à formação do ser humano, ao amadurecimento e à plenitude do indivíduo de forma gradual (TREVIZAN et al., 2010). Devem ser alicerçados por valores éticos, políticos, culturais e espirituais que estabeleçam conceitos multidisciplinares e globais. Para o alcance da multidisciplinaridade, os processos educativos necessitam de estratégias que priorizem o envolvimento profissional e pessoal do indivíduo, habilitando-o para o mundo do trabalho, num 
caminho de construção e reconstrução dinâmico e inovador (PASCHE, 2009; PASCHOAL, MANTOVANI \& MÉIER, 2007), capaz de conferir competências e entrega de serviços qualificados. Desse modo, seguindo as tendências das grandes organizações, as instituições de saúde passaram a incorporar os conceitos de aprendizagem, competência e gestão de conhecimento (ROCHA et al., 2012; SHINYASHIKI, TREVIZAN \& MENDES, 2003).

As universidades têm um importante papel na assistência, pesquisa e principalmente na formação de recursos humanos. Para obter sucesso e os melhores resultados, precisam de métodos pedagógicos que respondam aos cenários mundiais e transmitam valores humanos e éticos de forma coerente, coexistente e interativa (PASCHE, 2009; PASCHOAL, MANTOVANI \& MÉIER, 2007; DAL SASSO \& SOUZA, 2006). Para atingir tais fins, têm sido bastante explorados as metodologias e os métodos que envolvem o aprendiz com seu aprendizado, entre os quais se destaca a aprendizagem significativa.

A aprendizagem significativa requer a participação ativa do aluno nas estratégias de ensino utilizadas. É caracterizada pela interação cognitiva entre o novo conhecimento e o conhecimento prévio do estudante. Nesse processo, o novo conhecimento incorpora significados para $\mathrm{O}$ aprendiz, e o conhecimento prévio se torna mais rico, diferenciado e elaborado, adquirindo maior estabilidade. Por meio desse método, o aprendiz não é um receptor passivo: ele constrói, reconstrói, produz seu conhecimento e aprende com significado (AUSUBEL, 1982; MOREIRA, 2005). Para que a aprendizagem significativa ocorra, faz-se necessário ao estudante ser parte integrante do processo do novo conhecimento e ter disposição para o aprendizado. $\mathrm{O}$ conteúdo a ser aprendido deve ser potencialmente significativo, e na trajetória do estudante é preciso considerar os conhecimentos prévios, a vinculação dos conceitos existentes com os novos e a participação ativa dos sujeitos no processo de ensino-aprendizagem (PELIZZARI et al., 2002).

Nesse tipo de aprendizagem, as ideias mais gerais e inclusivas da matéria devem ser trabalhadas desde o início, progressivamente, com organização sequencial do conteúdo e consolidando os conceitos antes que os novos conhecimentos sejam apresentados. Os questionamentos e a interação social são as bases do relacionamento professor/ aluno, com o uso de uma grande diversidade de materiais instrucionais, cuidadosamente selecionados, que proporcionem ao aprendiz perceber o aprendizado, vivenciar a sua linguagem, observando que o significado está nas pessoas e que é necessário aprender a aprender, superar o erro e desaprender o que é irrelevante quando necessário (subsunçor ou o conhecimento prévio) (MOREIRA, 2005).

Nesse contexto, as Tecnologias de Informação e Comunicação (TICs) têm-se mostrado como respeitáveis ferramentas, uma vez que propiciam novas estratégias de se realizar o processo de ensino-aprendizagem. Vêm sendo aplicadas tanto na formação, como no aprimoramento e capacitação dos profissionais de saúde, com destaque para as estratégias de Educação a Distância $(\mathrm{EaD})$ e de Ensino Simulado.

A simulação é uma tentativa de imitar as particularidades de uma determinada situação clínica, ambicionando melhor compreensão e gestão dessa condição em contexto real. Recorre a um ambiente artificial para recriar uma situação real com o propósito de praticar, aprender, avaliar, testar e desenvolver a compreensão dos sistemas ou ações humanas. Aumenta e promove o crescimento de aprendizagens significativas e pode atingir o seu potencial máximo se os participantes a encararem como legítima, autêntica e real. É um processo de educação cognitiva e comportamental, que leva a altos níveis de autoestima e autoconfiança, ampliando a interiorização da informação e a satisfação com o processo de aprendizagem. Seu grande avanço está aliado ao uso de simuladores durante os treinos de pilotos na Segunda Guerra Mundial e ao desenvolvimento da cibernética. Nos dias atuais, impulsionada por questões de segurança, qualidade, ética, tecnologia e contextos da prática em constante mutação, tem se mostrado como imprescindível para o desenvolvimento e a formação dos profissionais de saúde (DAL SASSO 
\& SOUZA, 2006; MARTINS et al., 2012; LEIGH, 2008; CAMACHO, 2010).

A EaD é um sistema tecnológico, bidirecional, que pode ou não atingir um grande contingente de pessoas e que transfere a sala de aula de um espaço físico para um espaço virtual (OLIVEIRA, 2007). Entre as diversas estratégias utilizadas em $\mathrm{EaD}$, as relacionadas com os Ambientes Virtuais de Aprendizagem (AVA) e a videoconferência têm sido frequentes (SEIXAS et al., 2012).

A videoconferência é uma conexão entre pessoas, que pode ser realizada de forma individual ou coletiva, em tempo real e em posições remotas, com a finalidade de comunicação envolvendo áudio, texto e vídeo, fornecendo, no seu modo mais simples, a transmissão de imagens estáticas e texto entre duas posições e, no seu formato mais sofisticado, a transmissão de imagens em vídeo full-motion e áudio de alta qualidade entre posições múltiplas (SEIXAS et al., 2004). Os AVAs fazem parte do ciberespaço, incluem interfaces instrucionais, com foco na aprendizagem, e permitem a interação individual e/ou coletiva (RANGEL et al., 2011).

No Brasil, as evidências científicas demonstram o avanço do uso da simulação. No entanto, o custo para a implantação dos centros de simulação é oneroso, o que tem levado algumas instituições a confiarem que o "assistir" ou "participar" de práticas simuladas de diferentes complexidades por estratégias de $\mathrm{EaD}$ como a videoconferência podem ser substitutivos do laboratório simulado.

Nesse contexto, este estudo tem como objetivo comparar o resultado da aprendizagem de estudantes de graduação em enfermagem que utilizaram estratégias de ensino simulado de baixa fidelidade com o resultado do uso de videoconferência do ensino simulado de alta fidelidade.

\section{Metodologia}

Trata-se de um estudo experimental realizado numa universidade pública do interior do estado de São Paulo-Brasil, junto a estudantes do curso de Enfermagem.

Seguidos os preceitos éticos (Conep, Parecer n. 195/2011), este estudo foi desenvolvido durante a realização de um workshop sobre "Assistência de Enfermagem na Retenção Urinária". Todos os graduandos dos cursos de Bacharelado e Bacharelado e Licenciatura em Enfermagem do segundo ao quinto ano foram convidados. Entre os que aceitaram o convite, foram incluídos os que cumpriram todas as etapas propostas. Os estudantes desistentes, ou que não participaram de todas as etapas, foram excluídos. Utilizou-se o Termo de Consentimento Livre e Esclarecido.

Para o cumprimento do objetivo proposto, o estudo se desenvolveu em fase 1 e fase 2 .

$\mathrm{Na}$ fase 1, o conteúdo teórico foi disponibilizado através de estratégias de educação a distância (AVA/plataforma Moodle e videoconferência). Para a execução dessa etapa, foram realizados pelos pesquisadores: 1) a construção e ambientação da temática na plataforma; 2) a validação de aparência e conteúdo por juízes do material produzido. Considerou-se como aceitável o nível de concordância de 70\% (GRANT \& DAVIS, 1997).

Ainda na fase 1, após acesso ao AVA os estudantes participaram de uma videoconferência (V1), em que o conteúdo teórico foi discutido por especialistas. Na sequência, responderam a um questionário referente ao uso da videoconferência como estratégia para o ensino (ALEMÁN, GEA \& MODÉJAR, 2011).

$\mathrm{Na}$ fase 2, os estudantes foram divididos aleatoriamente em dois grupos (A e B), independente da fase de formação profissional e das experiências clínicas sobre o assunto estudado.

$\mathrm{O}$ grupo $\mathrm{A}$ realizou o treino em simulador de baixa fidelidade, e o grupo B participou de videoconferência de simulação de alta fidelidade com uso de simulador robótico e seu respectivo debriefing, sem, no entanto, fazer treino prático. $\mathrm{O}$ que define a complexidade ou fidelidade da atividade simulada é o objetivo de aprendizagem que o aprendiz deverá atingir. $\mathrm{O}$ treino simulado de baixa fidelidade implica a resolução de atividades pouco complexas que geralmente envolvem o treino de habilidades ou o desenvolvimento de competências específicas; nesse estudo o treino simulado de baixa fidelidade 
foi composto pela realização do cateterismo urinário em um simulador de baixa fidelidade (ou seja, num manequim de corpo inteiro que não permite nenhuma interação com o aprendiz). O treino simulado de alta fidelidade envolve o raciocínio clínico, a tomada de decisão, o uso de competências integradas, o trabalho em equipe, e geralmente está associado a cenários mais complexos (nesse estudo foi desenvolvido um cenário de alta fidelidade para assistência de enfermagem ao paciente com retenção urinária; nesse caso, foi utilizado um simulador de alta fidelidade, robótico).

Ao final da fase 2, os estudantes do grupo B responderam novamente ao questionário sobre $\mathrm{o}$ uso da videoconferência como estratégia para o ensino (ALEMÁN, GEA \& MODÉJAR, 2011).

Em seguida, para que fosse possível identificar o resultado da aprendizagem dos estudantes dos dois grupos, eles foram submetidos à avaliação teórica e teórico-prática.

Para a avaliação teórica, utilizou-se um teste de conhecimentos, com dez questões de múltipla escolha, incluindo os diferentes conteúdos abordados no workshop.Já a avaliação prática usou um instrumento de observação sistematizado, com as diversas ações que o estudante deveria desenvolver na prática, tendo como possibilidades "realiza", "não realiza" e "incompleto". Os instrumentos de coleta foram analisados por painel de peritos, com vista à sua validade de conteúdo e aparência, admitindo-se como aceitável o nível de concordância de 70,0\% (GRANT \& DAVIS, 1997).
A avaliação prática dos sujeitos foi realizada por um grupo de juízes, entre os quais os pesquisadores não faziam parte. Tais avaliadores foram treinados antes do processo de avaliação.

Os dados da pesquisa foram tabulados em planilhas do software Microsoft Excel, exportados e analisados no programa SAS/STAT (versão 9.0). A análise da percepção dos alunos com relação à videoconferência foi realizada através de estatística descritiva e do teste de concordância de Kappa.

Para a análise do aprendizado dos alunos, agruparam-se os dados em seis domínios, os primeiros cinco resultantes da avaliação prática e o último resultante da avaliação teórica: 1) interação, 2) avaliação de dados subjetivos, 3) avaliação de dados objetivos, 4) intervenção, 5) reavaliação e 6) avaliação teórica. Foram calculadas as estatísticas descritivas por domínio e por grupo e a estatística inferencial (t-Student) para comparar as médias entre os grupos A e B.

\section{Resultados}

Ao final do período disponibilizado para as inscrições, 140 estudantes estavam inscritos no AVA; no entanto, apenas 44 completaram todas as etapas, sendo $42(95,0 \%)$ do sexo feminino e dois $(5,0 \%)$ do sexo masculino. Os motivos de desistência apontados por grande parte dos sujeitos do curso foram as atividades presencias (avaliações) e a incompatibilidade de agenda. Os dados relacionados

\begin{tabular}{|l|l|c|}
\hline Atividade & & $\mathbf{N}^{*}$ \\
\hline \multirow{2}{*}{ Fase 1 } & Inscrição no AVA Moodle & 140 \\
\hline & Finalizaram o AVA Moodle & 53 \\
\hline & Participaram da videoconferência 1 & 47 \\
\hline \multirow{2}{*}{ Fase 2 } & Grupo A - Treino simulado de baixa fidelidade & 20 \\
\hline & Grupo B - Participaram da videoconferência 2 & 27 \\
\hline & Avaliação teórica & $\begin{array}{c}\text { Grupo A - 17 } \\
\text { Grupo B - 27 }\end{array}$ \\
\cline { 2 - 3 } & Avaliação prática & $\begin{array}{c}\text { Grupo A - 17 } \\
\text { Grupo B - 27 }\end{array}$ \\
\hline
\end{tabular}

Quadro 1 - Número de participantes nas etapas do estudo. *N: número de participantes. 
com o número de participantes em cada etapa das atividades estão descritos no Quadro 1.

\section{O AVA Moodle}

As atividades foram disponibilizadas em módulos gradativamente no AVA e ficaram disponíveis para acesso dos estudantes durante treze dias. Os estudantes que cumpriram todas as atividades utilizaram em média oito dias. O estudante que realizou as atividades em maior período de tempo usou treze dias, e o que fez em menor prazo precisou de cinco dias. Apenas os estudantes que fizeram acessos ao AVA no período de disponibilização do módulo final tiveram oportunidade de concentrar os acessos em menor número de dias.

Os períodos escolhidos para a realização das atividades foram, pela maior parte dos estudantes, o vespertino e o matutino, e o tempo utilizado teve duração aproximada de quinze minutos. Mesmo após o término das atividades, foram registrados 331 acessos dos estudantes ao AVA.

\section{Videoconferências 1 e 2}

Todos os alunos na videoconferência 1 e na videoconferência 2 informaram que a aula atingiu os objetivos propostos e que auxiliou o aprendizado. O Quadro 2 apresenta a percepção dos alunos quanto às videoconferências 1 (V1) e 2 (V2).

Entre os 27 (100,0\%) estudantes do grupo B que participaram de videoconferência 2, 25 (92,6\%) relataram que participar da videoconferência com uso de simulador de alta fidelidade e seu respectivo debriefing reforçou o aprendizado. Os motivos apontados foram: onze $(44,0 \%)$ - que a simulação aproxima o estudante da prática do enfermeiro; seis $(24,0 \%)$ que promove o aumento da segurança; seis $(24,0 \%)$ - que o evento foi motivador e inovador; dois $(8,0 \%)$ - que essa é uma nova estratégia de ensino. Dentre os estudantes, dois mencionaram que assistir ao treino da videoconferência não auxiliou no seu aprendizado, um justificou o fato pelo áudio ruim.

Houve altas porcentagens de concordância da avaliação quanto às videoconferências 1 e 2: relativamente a ter sido boa a interação (Kappa $=1$ ) e aos fatos de o recurso ter auxiliado no aprendizado $($ Kappa $=1)$, de o conteúdo e a curiosidade haverem motivado a participação na aula por videoconferência (Kappa $=1$ ), e de a aula ter atingido os objetivos propostos (Kappa $=1$ ). Em relação à avaliação da concentração nas

\section{Percepção dos alunos}

\section{Concentração}

$\operatorname{Sim}$

Não

\section{Interação}

Boa

Outro

\section{Fator de motivação}

Conteúdo

Estratégia inovadora

Curiosidade

Outro

\section{Videoconferência 1 Grupos A e B}

$45(95,74 \%)$

$2(4,26 \%)$

\section{Videoconferência2 Grupo B}

$$
24(88,89 \%)
$$

$3(11,1 \%)$

$44(93,6 \%) \quad 27(100,0 \%)$

$3(6,4 \%)$

$0(0 \%)$

Quadro 2 - Percepção dos alunos quanto às videoconferências 1 e 2 como ferramentas para o ensino. 
videoconferências, houve concordância moderada (Kappa $=0,47)$.

\section{Aprendizado dos estudantes dos grupos A e B}

Conforme demonstra a Tabela 1, o aprendizado dos estudantes foi agrupado em seis domínios e comparado entre os grupos A e B. O grupo A apresentou médias mais elevadas na avaliação da aprendizagem, com significância no domínio avaliação de dados objetivos e no domínio avaliação teórica.

A Tabela 2 apresenta o aprendizado dos alunos segundo sua experiência teórica, prática ou em laboratório anterior à realização do estudo em que os valores médios positivos foram superiores aos valores médios negativos nos dois grupos e nos três contextos.

\section{Discussão}

Devido à ausência de encontros presenciais, à solidão e à falta de interação entre estudantes e professores e entre os próprios estudantes, as estratégias de ensino a distância $(\mathrm{EaD})$ ainda possuem baixa adesão e alta evasão entre estudantes, embora tenham uma excelente amplitude de alcance. Para minimizar tal situação, recomenda-se que durante as atividades sejam utilizadas ferramentas como chats, fóruns, e-mails ou encontros presenciais, pois reforçam o interesse do estudante e contribuem para o sucesso do processo de ensino-aprendizagem (FÁVERO \& FRANCO, 2006; FILHO \& OLIVEIRA, 1999). Nesta pesquisa, dentre os inscritos, cerca de um terço dos estudantes não concluíram as atividades do AVA Moodle, alegando como motivo a impossibilidade de participação nas atividades presenciais, o que reforça a importância da liberdade de locais de acesso e horários para a realização das atividades de $\mathrm{EaD}$. Entre os que concluíram, não houve dificuldades de acesso, o que pôde ser verificado pelo tempo destinado às atividades e pelo interesse manifestado através dos frequentes acessos à plataforma mesmo após o término do workshop.

Com relação às videoconferências, embora quase todos os estudantes do grupo B tenham

\begin{tabular}{|c|c|c|c|c|c|}
\hline Domínio & Grupo & Média & $p$ valor* & \multicolumn{2}{|c|}{$I C^{* *}(95 \%)$} \\
\hline Interação & $\begin{array}{l}\text { A } \\
B\end{array}$ & $\begin{array}{l}67,6 \\
62,0\end{array}$ & 0,461 & $-9,515$ & 20,735 \\
\hline Avaliação: dados subjetivos & $\begin{array}{l}A \\
B\end{array}$ & $\begin{array}{l}34,6 \\
24,3\end{array}$ & 0,246 & $-7,334$ & 28,056 \\
\hline Avaliação: dados objetivos & $\begin{array}{l}\text { A } \\
\text { B }\end{array}$ & $\begin{array}{c}47,1 \\
26,4\end{array}$ & 0,018 & 3,538 & 37,802 \\
\hline Intervenção & $\begin{array}{l}\text { A } \\
B\end{array}$ & $\begin{array}{l}40,5 \\
38,7\end{array}$ & 0,786 & $-11,650$ & 15,329 \\
\hline Reavaliação & $\begin{array}{l}A \\
B\end{array}$ & $\begin{array}{l}43,1 \\
39,5\end{array}$ & 0,699 & 15,079 & 22,341 \\
\hline Teórico & $\begin{array}{l}\text { A } \\
\text { B }\end{array}$ & $\begin{array}{l}68,7 \\
51,8\end{array}$ & 0,006 & 4,994 & 28,803 \\
\hline Geral & $\begin{array}{l}\text { A } \\
B\end{array}$ & $\begin{array}{l}46,7 \\
36,9\end{array}$ & 0,078 & $-1,143$ & 20,762 \\
\hline
\end{tabular}

Tabela 1 - Aprendizado por domínio e grupo. * $p$ valor: significância do teste; **IC: intervalo de confiança. 


\begin{tabular}{|lllll|}
\hline Experiência Teórica & Grupo & N & Média & $\begin{array}{l}\text { Desvio } \\
\text { Padrão }\end{array}$ \\
\hline Negativa & A & 4 & 32,9 & 15,8 \\
\hline Positiva & B & 9 & 27,8 & 16,4 \\
\hline Experiência em Laboratório & A & 13 & 51,0 & 20,1 \\
\hline Negativa & B & 18 & 41,5 & 18,4 \\
\hline Positiva & & & & \\
\hline Experiência prática & A & 4 & 36,8 & 19,3 \\
\hline Negativa & B & 15 & 38,0 & 16,4 \\
\hline Positiva & A & 12 & 51,5 & 19,6 \\
& B & 12 & 35,5 & 18,5 \\
\hline
\end{tabular}

Tabela 2 - Aprendizado em relação às experiências anteriores.

percebido a videoconferência 2 como uma estratégia "inovadora e motivante" que reforçou o seu aprendizado e os aproximou da prática clínica do enfermeiro, aumentando sua segurança, isso não se refletiu nos resultados da avaliação de aprendizagem.

O enorme potencial da videoconferência resulta da junção de áudio e vídeo e da comunicação entre professores e estudantes. No entanto, quando o processo de formação envolve práticas laboratoriais, é de extrema importância um sistema de áudio e vídeo de grande qualidade, que possa dar visibilidade às práticas simuladas, com precisão e apresentação de detalhes, garantindo ao estudante maior concentração e foco na atividade (GODOY et al., 2004; SALVADOR et al., 2010). Durante a videoconferência 2 houve problemas no áudio, o que pode ter afetado o nível de concentração e ocasionado limitações ao estudo.

As mudanças ocorridas nos paradigmas de ensino/formação colocam, atualmente, o estudante no centro da aprendizagem, elevando consideravelmente o uso da simulação e particularmente da simulação de alta fidelidade. $\mathrm{Na}$ formação dos profissionais da área da saúde, a simulação cria em ambiente controlado uma determinada situação clínica, ambicionando uma melhor compreensão e gestão dessa situação em contexto real. É um método de aprendizagem ativa que reforça os conhecimentos teóricos adquiridos em sala de aula e, por refletir a realidade, contribui para a participação do estudante na sua própria aprendizagem (AUSUBEL, 1982; HAWKINS, TODD \& MANZ, 2008; SCHIAVENATO, 2009). A prática simulada não é sinônimo do uso de tecnologias e recursos onerosos: trata-se de uma técnica que deve aproximar com a maior veracidade possível os estudantes da prática clínica na qual estarão inseridos. O que determina os recursos utilizados nas práticas simuladas são os objetivos almejados no processo de ensino-aprendizagem, os quais, nas mais variadas situações, contam com a expertise e a criatividade do docente, chamado 
nesse processo de facilitador. $\mathrm{O}$ facilitador possui papel preponderante na condução da atividade simulada e deve ter ainda o domínio dos atributos tecnológicos, assistenciais e didáticos para a condução do método.

Nesse estudo, ao compararmos o desempenho dos grupos A e B, é possível observar que os estudantes do grupo A (submetidos à simulação de baixa fidelidade) foram melhor avaliados do que os estudantes do grupo B (que participaram da videoconferência com uso de simulador de alta fidelidade e seu respectivo debriefing), sendo significativas as diferenças entre os grupos nos domínios classificados: avaliação dos dados objetivos e avaliação teórica. Tais resultados demonstram a importância do fazer nos aspectos cognitivos, no treino de habilidades e no desenvolvimento de competências durante a formação dos profissionais da área de saúde. Além disso, os números obtidos incentivam ainda o uso dos laboratórios didáticos como recurso expressivo no processo de ensino-aprendizagem e reforçam a tese de que em simulação é necessário vivenciar as práticas propostas. $\mathrm{O}$ treino simulado promove o desenvolvimento de aprendizagens significativas e demonstra eficácia na educação cognitiva e comportamental (HOADLEY, 2009).É a oportunidade para o estudante vivenciar a prática clínica da profissão e participar da construção do seu aprendizado, num contexto seguro.

Destacamos como fatores limitantes desse estudo os fatos de que o número de participantes ao longo do trabalho e também seus resultados podem ter sido influenciados pela dispersão dos estudantes devido à estratégia de ensino inovadora e/ou à dificuldade apresentada no recurso de áudio na videoconferência.

\section{Conclusão}

Conforme os resultados encontrados, o uso das Tecnologias de Comunicação e Informação (TICs) no ensino, entre as quais se incluem as diferentes estratégias de $\mathrm{EaD}$ e simulação, desperta o interesse do estudante e pode ser considerado atual e interativo, pois parte de um contexto em que o aprendiz é participante ativo do seu processo de ensino-aprendizagem.

Embora limitado pelas dificuldades de áudio, esse estudo mostrou melhor desempenho dos estudantes submetidos à simulação de baixa fidelidade quando comparados com os estudantes submetidos a estratégias de ensino com uso da videoconferência do treino simulado de alta fidelidade e seu respectivo debriefing. Nesse contexto, suscita, entre outras, novas investigações que avaliem os diferentes níveis de aprendizado de estudantes que participam em grupo nos ambientes simulados.

A simulação é um método de aprendizagem significativo que requer a participação efetiva do estudante. O desenvolvimento de atividades simuladas, em cenários de baixa, média ou alta fidelidade, é eficaz e propicia ao estudante aprender com significado.

\section{Referências Bibliográficas}

ALEMÁN, J. L. F.; GEA, J. M. C. \& MODÉJAR, J. J. R. "Effects of Competitive Computer-Assisted Learning Versus Conventional Teaching Methods on the Acquisition and Retention of Knowledge in Medical Surgical Nursing Students". Nurse Education Today, vol. 31, n. 11, pp. 866-871, 2011.

AUSUBEL, D. P. A Aprendizagem Significativa: A Teoria de David Ausubel. São Paulo: Moraes, 1982.

CAMACHO, H. M. Simulación Cibernética en las Ciencias de la Salud. Recuento Histórico en el Mundo y en Colombia y su Impacto en la Educación. 2. ed. Bogotá, Colômbia: Kimpres, 2010.

DAL SASSO, G. T. M. \& SOUZA, M. L. "Computer-Assisted Simulation: Convergence in the Process of Education and Care in Nursing". Texto \& Contexto Enfermagem, vol. 15, n. 2, pp. 231-239, 2006.

FÁVERO, R. V. M. \& FRANCO, S. R. K. "Um Estudo sobre a Permanência e a Evasão na Educação a Distância”. Novas Tecnologias na Educação, vol. 4, n. 2, pp. 1-10, 2006.

FILHO, G. O. M. \& OLIVEIRA, A. M. B. "Invente uma Investigação do Ensino Tecnológico à Distância". Revista Educação \& Tecnologia, n. 5, pp. 62-72, 1999.

GODOY, S.; MENDES, I. A. G.; HAYASHIDA, M.; NOGUEIRA, M. S. \& ALVES, L. M. M. "In-Service Nursing Education Delivered by Videoconference". Fournal of Telemedicine and Telecare, vol. 10, n. 5, pp. 303-305, 2004.

GRANT, J. S. \& DAVIS, L. L. "Selection and Use of Content Expert for Instrument Development". Research in Nursing \& Health, vol. 20, n. 3, pp. 269-274, 1997. 
HAWKINS, K.; TODD, M. \& MANZ, J. "A Unique Simulation Teaching Method". Fournal of Nursing Education, vol. 47, n. 11, pp. 524-527, 2008.

HOADLEY, T. A. "Learning Advanced Cardiac Life Support: A Comparison Study of the Effects of Low and High-Fidelity Simulation". Nursing Education Perspectives, vol. 30, n. 2, pp. 91-97, 2009.

LEIGH, G.T. "High-Fidelity Patient Simulation and Nursing Students Self-Efficacy: A Review of Literature". International fournal of Nursing Education Scholarship, vol. 5, n. 1, pp. 1-16, 2008.

MARTINS, J. C. A.; MAZZO, A.; BAPTISTA, R. C. N.; COUTINHO, V. R. D.; GODOY, S.; MENDES, I. A. C. \& TREVIZAN, M. A. "The Simulated Clinical Experience in Nursing Education: A Historical Aproach". Acta Paulista de Enfermagem, vol. 25, n. 4, pp. 619-625, 2012.

MOREIRA, A. M. "Aprendizagem Significativa Crítica". Indivisa, Boletín de Estudios e Investigación, vol. 1, n. 6 , pp. 83-101, 2005.

OLIVEIRA, M. A. N. "Distance Education as Strategy for Permanent Education in Health: Possibilities and Challenges". Revista Brasileira de Enfermagem, vol. 60, n. 5, pp. 585-589, 2007.

PASCHE, D. F. "National Humanization Policy as a Commitment to Collective Production of Changes in Management and Care Methods". Interface - Comunicaşão, Saúde, Educaşão, n. 13, Suppl. 1, pp. 701-708, 2009.

PASCHOAL, A. S.; MANTOVANI, M. F. \& MÉIER, M. J. "The Perception of Permanent, Continuous, in Service Education for Nurses in a School Hospital". Revista da Escola de Enfermagem da USP, vol. 41, n. 3, pp. 478-484, 2007.

PELIZZARI, A.; KRIEGL, M. L.; BARON, M. P.; FINCK, N. T. L. \& DOROCINSKI, S. I. "Teoria da Aprendizagem Significativa segundo Ausubel". Revista do Programa de Educasão Corporativa, vol. 2, n. 1, pp. 37-42, 2002.
RANGEL, E. M.; MENDES, I. A. C.; CARNIO, E. G.; ALVES, L. M. M.; GRISPIM, J. A.; MAZZO, A.; ANDRADE, J. X.; TREVIZAN, M. A. \& RANGEL, A. L. "Evaluation by Nursing Students in Virtual Learning Environments for Teaching Endocrine Physiology". Acta Paulista de Enfermagem, vol. 24, n. 3, pp. 327-33, 2011.

ROCHA,E.S.B.;NAGLIATE,P.;FURLAN, C.E.B.; KERSON, Rocha Jr.; TREVIZAN, M. A. \& MENDES, I. A. C. "Knowledge Management in Health: A Systematic Literature Review". Revista Latino-Americana de Enfermagem, vol. 20, n. 2, pp. 392-400, 2012.

SALVADOR, M. E.; MOREIRA, R. S.; HIROMI, L.T.; PEREIRA, S. R. \& CARMAGNANI, M. I. S. "Using Videoconferencing to Discuss Themes of Nursing Management in University Hospitals". Acta Paulista de Enfermagem, vol. 23, n. 5, pp. 705-708, 2010.

SCHIAVENATO, M. "Reevaluating Simulation in Nursing Education: Beyond the Human Patient Simulator". Fournal of Nursing Education, vol. 48, n. 7, pp. 388-394, 2009.

SEIXAS, C. A.; MENDES, I. A. C.; GODOY, S. \& COSTA, A. L. "Implantation of a Videoconferencing System Applied to Nursing Research and Teaching Environments". Revista Brasileira de Enfermagem, vol. 57, n. 5, pp. 620-624, 2004.

. et al. "Ambiente Virtual de Aprendizagem: Estruturação de Roteiro para Curso On-line". Rev. Bras. Enferm., vol. 65, n. 4, pp. 660-666, 2012.

SHINYASHIKI, G. T.; TREVIZAN, M.A. \& MENDES, I. A. C. "Sobre a Criação e a Gestão do Conhecimento Organizacional". Revista Latino-Americana de Enfermagem, vol. 11, n. 4, pp. 499-506, 2003.

TREVIZAN, M. A.; MENDES, I. A. G.; MAZZO, A. \& VENTURA, G. A. A. "Investment in Nursing Human Assets: Education and Minds of the Future". Revista Latino-Americana de Enfermagem, vol. 18, n. 3, pp. 467-471, 2010. 